\title{
Perfeccionismo e Representação Vinculativa em Jovens Adultos
}

\author{
Perfectionism and Attachment Representations in Young Adults
}

\author{
Diana Fernandes Oliveira*, Cláudia Carmo, José Pestana Cruz \& Marta Brás \\ Universidade do Algarve, Faro, Portugal
}

\begin{abstract}
Resumo
As relações estabelecidas no seio familiar são extremamente importantes no desenvolvimento dos traços de personalidade dos indivíduos. O objectivo deste estudo foi investigar a relação entre o desenvolvimento do perfeccionismo e as representações de vinculação, numa amostra de 690 jovens adultos, com idades entre os 17 e os 30 anos. Os participantes preencheram um Questionário Sócio-Demográfico, duas Escalas Multidimensionais de Perfeccionismo (MPS-F e MPS-H) e um Inventário de Vinculação (IPPA). Os resultados indicam a existência de uma relação entre as dimensões maladaptativas do perfeccionismo e a representação de uma Vinculação Insegura à mãe e ao pai, enquanto as dimensões adaptativas do perfeccionismo relacionam-se com a representação de uma Vinculação Segura. Estes resultados sugerem que um dos factores que contribuem para o desenvolvimento do perfeccionismo é a natureza das relações parentais e consequentes interacções.
\end{abstract}

Palavras-chave: Perfeccionismo, representações de vinculação, vinculação insegura, vinculação segura.

\begin{abstract}
The relationships established within the family are extremely important in the development of individual personality traits. The goal of this study was to investigate the relation between the development of perfectionism and attachment representations in a sample of 690 young adults, aged between 17 and 30 years old. Participants completed two scales of Self-report Measure of Perfectionism (H-MPS and F-MPS) and the Inventory of Parent and Peer Attachment (IPPA), as well as a Social-Demographic Questionnaire. Results indicate the existence of a relation between maladaptive perfectionism dimensions and an insecure attachment representation to parents, whereas adaptive perfectionism dimensions seem to be related to a secure attachment representation. The results suggest that one of the contributing factors to the development of perfectionism is the nature of parental relationships and their consequent interactions.

Keywords: Perfectionism, attachment representations, insecure attachment, secure attachment.
\end{abstract}

O perfeccionismo tem sido definido como a tendência para estabelecer padrões excessivamente elevados, pela rígida adesão a esses mesmos padrões, e pelas auto-avaliações demasiado críticas (Frost, Marten, Lahart, \& Rosenblate, 1990; Shafran \& Mansell, 2001). Uma miríade de estudos tem comprovado a influência do perfeccionismo em diversas perturbações psicológicas, tais como depressão (e.g., Blatt, 1995; Sherry, Hewitt, Flett, \& Harvey, 2003), insónia (e.g., Azevedo et al., 2008), perturbações da ansiedade (e.g., Stoeber, Feast, \& Hayword, 2009; Wu \& Cortesi, 2009), e perturbações do comportamento alimentar (e.g., Bardone-Cone, Sturm, Lawson, Robinson, \& Smith, 2010). A presença do perfeccionismo num destes quadros psicopatológicos contribui para uma maior severidade dos sintomas, para tratamentos mais demorados e para uma maior probabilidade de recidivas (Cruz, 2009).

\footnotetext{
${ }^{*}$ Endereço para correspondência: Departamento de Psicologia, Faculdade de Ciências Humanas e Sociais, Universidade do Algarve, Campus de Gambelas, Faro, Portugal 8005-139. E-mail: dianafernandesoliveira@gmail.com
}

Segundo alguns autores (e.g., Burns, 1980; Soenens, Vansteenkiste, Luyten, Duriez, \& Goossens, 2005) o perfeccionismo é um traço de personalidade disfuncional e desadaptativo. Contudo, Hamachek (1978) postulou que o perfeccionismo pode ser normal e que o esforço associado ao desejo de atingir a perfeição pode conduzir a um ajustamento positivo. Hamachek (1978) foi um dos primeiros autores a realçar a necessidade e a importância da distinção entre perfeccionismo normal ou adaptativo e perfeccionismo neurótico ou maladaptativo. O perfeccionismo normal ou adaptativo como o esforço para atingir padrões razoáveis e realistas, que quando atingidos conduzem a um sentimento de auto-satisfação e ao aumento da auto-estima, em vez de diminuí-la. Os perfeccionistas normais são descritos como indivíduos que definem objectivos elevados e são capazes de estabelecer limites à sua performance tendo em consideração as suas forças e limitações, conseguindo retirar prazer dos trabalhos que exigem grandes esforços e sentindo-se livres para serem menos precisos conforme a situação o permita (Hamachek, 1978; Rice, Lopez, \& Vergara, 2005). Por outro lado, o perfeccionismo neurótico abrange uma 
preocupação excessiva em cometer erros e uma incapacitante culpabilização do self, excessiva auto-crítica, um sentimento persistente de que os padrões ou expectativas auto-impostos não estão a ser alcançados e uma preocupação em desiludir os outros (Hamachek, 1978).

Neste sentido, as investigações têm confirmado a hipótese de que o perfeccionismo é um construto multidimensional, com aspectos adaptativos e maladaptativos, podendo ter um impacto negativo na vida dos adolescentes e jovens adultos (e.g., Frost et al., 1990; Hewitt \& Flett, 1990, 1991). Segundo Craddock, Church e Sands (2008), o perfeccionismo abrange a tendência para estabelecer padrões excessivamente elevados, aderindo rigidamente aos mesmos, a valorizar fortemente a obtenção de excelência e a perscrutar os resultados de uma forma extremamente crítica. Os autores salientam que esta tendência é comummente associada aos esforços dos adolescentes e jovens adultos para obter uma performance excelente ao nível educacional, sendo que o seu impacto poderá ser negativo. Enns, Cox, Sareen e Freeman (2001) referem que o perfeccionismo maladaptativo pode interferir com a performance social e académica dos estudantes. Neste contexto, Wei, Mallinckrodt, Russell e Abraham (2004) revelam a existência de uma associação positiva entre o perfeccionismo e a depressão ou o desespero, destacando alguns estudos com estudantes universitários onde se verificou uma associação entre o perfeccionismo e sintomatologia depressiva (e.g., Chang, 2002; Chang \& Sana, 2001) e preocupação suicida (e.g., Adkins \& Parker, 1996; Chang, 1998).

Nas últimas décadas diversos autores (e.g., Craddock et al., 2008; Frost, Lahart, \& Rosenblate, 1991) têm especulado sobre as origens do perfeccionismo, dirigindo a sua atenção para a natureza das relações pais-filho e consequentes interacções (e.g., Barrow \& Moore, 1983; Burns, 1980; Camadan, 2010; Craddock et al., 2008; Kawamura, Frost, \& Harmatz, 2002; Rice, Ashby, \& Preusser, 1996; Rice et al., 2005) sugerindo que a origem do perfeccionismo está relacionada com uma "vinculação problemática" (Wei et al., 2004, p. 202). Bowlby (1977) definiu a vinculação como sendo a "propensão do ser humano para estabelecer fortes ligações afectivas com outros significativos" (Platts, Tyson, \& Mason, 2002, p. 332), defendendo também que a acessibilidade de figuras parentais seria o único meio capaz de produzir sentimentos de segurança na criança (V. F. Gomes \& Bosa, 2010).

A vinculação segura surge quando os pais são emocionalmente acessíveis e carinhosos com os seus filhos. Este tipo de vinculação fornece uma sensação de conforto e previsibilidade, encorajando a abordagem de novos desafios interpessoais e desenvolvimentais (Rice $\&$ Mirzadeh, 2000). Por seu turno, a vinculação insegura resulta de relações pais-criança imprevisíveis, severas e instáveis. As crianças com vinculações inseguras têm dificuldades em gerir desafios desenvolvimentais e experienciam uma grande diversidade de problemas de ajus- tamento pessoais e interpessoais (Bowlby, 1973, citado por Rice \& Mirzadeh, 2000). Sochos e Tsalta (2008) salientam que os indivíduos com uma vinculação insegura generalizam acerca do self e dos outros, com base nas experiências negativas com os prestadores de cuidados e parceiros íntimos. Estes indivíduos desenvolvem distorções cognitivas que reforçam a vinculação insegura com os outros, as relações interpessoais não sustentadoras e a sintomatologia depressiva.

Vários autores postulam que a ideia de se ser perfeito é influenciada por experiências precoces vivenciadas no ambiente familiar, e, que experiências com estilos parentais disfuncionais e com características disfuncionais do sistema familiar estão associadas com a manifestação de perfeccionismo maladaptativo nos jovens adultos (e.g., Craddock et al., 2008; Frost et al., 1991; Kawamura et al., 2002; Rice et al., 1996). Gamble e Roberts (2005) sugerem que cognições negativas acerca do self tais como crenças de auto-desvalorização e inutilidade, estão associadas com percepções negativas acerca da relação pais-criança. Num estudo com 134 adolescentes, estes autores verificaram que os filhos que referem que os pais efectuavam frequentemente comentários críticos e humilhantes acerca de si próprios ou que esperavam que sobressaíssem em todos os aspectos das suas vidas, tinham fortes preocupações acerca de um potencial abandono, apresentando, também, desconforto em aproximarse dos outros. Ainda neste estudo, a Vinculação Insegura encontrava-se directamente associada com uma baixa auto-estima, atitudes disfuncionais e um estilo atributivo negativo, onde as relações de interacção parental são diferentes quanto ao género dos adolescentes. As adolescentes demonstraram ser mais sensíveis aos efeitos da percepção de uma parental adversa.

Uma das formas de avaliação da vinculação em adolescentes e jovens adultos, apesar das limitações apontadas (Halverson, 1988; Laghi, D’Alessio, Pallini, \& Baiocco, 2009) passa pelo recurso a instrumentos de auto-relato, tendo sido especificamente desenvolvidos para avaliar estilos de vinculação e explorar a relação entre estes e a qualidade das trajectórias desenvolvimentais (Faria, 2008). Salientamos o Inventory of Parent and Peer Attachment ([IPPA], Armsden \& Greenberg, 1987), um instrumento adaptado e validado para a população portuguesa por Neves, I. Soares e Silva (1999) e utilizado no presente estudo. O IPPA tem sido aplicado em várias investigações sobre a vinculação nos adolescentes e jovens adultos (e.g., Cunha, I. Soares, \& Pinto-Gouveia, 2008; Geada, 1990; Mota \& Matos, 2009; Rice et al., 1996), permitindo a avaliação independente da qualidade de vinculação (segura/insegura) à mãe, ao pai e aos amigos, através de três factores: Confiança (abarca a compreensão parental, respeito e confiança mútua); Comunicação (qualidade da comunicação verbal com os pais) e Alienação (desvinculação e isolamento na relação). 
Apesar de diversos autores sugerirem que a origem do perfeccionismo está relacionada com uma "vinculação problemática" (Wei et al., 2004, p. 202) na relação parental, até há relativamente pouco tempo eram parcas as investigações empíricas sobre o perfeccionismo e a vinculação. Uma investigação com estudantes universitários, revelou que o perfeccionismo maladaptativo estava associado a uma vinculação insegura, enquanto o perfeccionismo adaptativo se relacionava com uma vinculação segura (Rice \& Mirzadeh, 2000).

O presente estudo tem como principais objectivos: investigar a relação entre os níveis de perfeccionismo e as representações de vinculação nos jovens adultos e averiguar a existência de uma relação entre as representações de vinculação e as diferentes dimensões de perfeccionismo apresentadas por Frost et al. (1990) e Hewitt e Flett (1991). Perante os objectivos propostos, procuramos, por um lado, perceber se existem diferenças estatisticamente significativas entre as representações de vinculação e as dimensões adaptativas e maladaptativas, formuladas por Frost et al. (1990) e Hewitt e Flett (1991), e, por outro, qual a relação existente entre os três factores que contribuem para o desenvolvimento das representações de vinculação (Confiança, Comunicação e Alienação), com as diversas dimensões de perfeccionismo, de Frost et al. (1990) e Hewitt e Flett (1991).

\section{Método}

\section{Participantes}

Os participantes foram seleccionados por conveniência, atendendo ao facto de serem adolescentes e jovens adultos, estudantes do distrito de Faro. A amostra é composta por 690 indivíduos $(N=690)$, com idades compreendidas entre os 17 e os 30 anos $(M=19,32 ; D P$ $=4,16)$, dos quais 442 são do género feminino $(64,1 \%)$ e 248 são do género masculino (35,9\%). A maioria dos participantes deste estudo é de nacionalidade portuguesa $(91,4 \%)$, com um grau de instrução ao nível do ensino universitário $(48,0 \%)$, secundário $(33,2 \%)$ e profissional $(10,7 \%)$, sendo provenientes de várias unidades orgânicas.

\section{Instrumentos}

Dados Sócio-Demográficos. Foi utilizado um Questionário Sócio-Demográfico, cujo objectivo é o de recolher informações pessoais, sociais e demográficas dos participantes.

Perfeccionismo. Para medir os níveis de perfeccionismo dos participantes, foram utilizadas duas escalas de avaliação: a Multidimensional Perfectionism Scale (MPS-F) de Frost et al. (1990), e a Multidimensional Perfectionism Scale (MPS-H) de Hewitt e Flett (1991), versão portuguesa (M. J. Soares, A. A. Gomes, Macedo, \& Azevedo, 2003). As duas escalas foram utilizadas em conjunto uma vez que partilham uma concepção multidimensional do constructo do perfeccionismo, no entan- to, a Multidimensional Perfectionism Scale (MPS-F) de Frost et al. (1990) possibilita ainda uma avaliação mais detalhada das componentes desenvolvimentais implicadas no perfeccionismo, avaliadas de acordo com estes autores pelas dimensões Expectativas Parentais e Críticas Parentais.

A Multidimensional Perfectionism Scale (Frost et al., 1990) é um instrumento composto por 35 itens, avaliados numa escala de tipo Likert de 5 pontos $(1=$ discordo completamente; 5 = concordo completamente), que mede seis dimensões de perfeccionismo (Frost et al., 1990): Preocupação Com os Erros (PCE), Padrões Pessoais (PP), Expectativas Parentais (EP), Críticas Parentais (CP), Dúvidas sobre as Acções Pessoais (DSAP) e Organização $(\mathrm{O})$. A título de exemplo, podemos considerar os seguintes itens: Preocupação Com os Erros - item 9: "Se falhar no meu trabalho/na escola, falho enquanto pessoa"; Padrões Pessoais - item 4: "Se eu não estabelecer os padrões mais elevados para mim, provavelmente acabarei como uma pessoa de segunda categoria"; Expectativas Parentais - item 1: "Os meus pais estabeleciam padrões muito elevados para mim"; Críticas Parentais - item 3: "Quando era criança, era punido quando não fazia as coisas de forma perfeita"; Dúvidas Sobre as Acções Pessoais - item 17: "Mesmo quando faço alguma coisa muito cuidadosamente, sinto com frequência que não a fiz suficientemente bem"; e, Organização - item 2: “A organização é muito importante para mim". A Escala Multidimensional de Perfeccionismo de Frost et al. (1990) revela uma boa consistência interna, uma vez que os coeficientes do alfa de Cronbach das sub-escalas variam entre 0,77 e $0,93(\mathrm{PCE}=0,88 ; \mathrm{PP}=0,83 ; \mathrm{EP}=0,84$; $\mathrm{CP}=0,84 ; \mathrm{DSAP}=0,77 ; \mathrm{O}=0,93)$ e o coeficiente da escala completa é de 0,90 (Frost et al., 1990; Rice \& Mirzadeh, 2000; Rice et al., 2005). No presente estudo procedeu-se à tradução da escala MPS-F, de forma a ser possível a sua aplicação. Esta tradução foi efectuada segundo as indicações de Pais-Ribeiro (2008), tendo-se verificado que não existiam discrepâncias assinaláveis relativamente à sua estrutura e conteúdo. Foi possível identificar uma solução de 6 factores, tal como proposto por Frost et al. (1990) na versão original. Os 6 factores explicaram 54,98\% da variância total. A análise da consistência interna mostrou um alfa de Cronbach de 0,87 para a pontuação total e valores também satisfatórios para as diferentes subescalas. A fiabilidade teste-reteste sugeriu uma boa estabilidade temporal da pontuação total da escala e das subescalas.

A Escala Multidimensional de Perfeccionismo- $H$ (Hewitt \& Flett, 1991; adaptação de M. J. Soares et al., 2003) é composta por 45 itens, avaliados numa escala de tipo Likert de 7 pontos, em que 1 corresponde a "discordo completamente" e 7 corresponde a "concordo completamente". Esta escala avalia três dimensões de perfeccionismo: perfeccionismo auto-dirigido, perfeccionismo socialmente prescrito e perfeccionismo dirigido aos outros. A título de exemplo, podemos considerar 
os seguintes itens: Perfeccionismo Auto-Orientado (PAO) - item 15: "Preocupo-me muito em ter um resultado perfeito em tudo o que faço"; Perfeccionismo Socialmente Prescrito (PSP) - item 31: "Sinto que as outras pessoas exigem demais de mim"; e, Perfeccionismo Dirigido aos Outros (PDO) - item 16: "Espero muito das pessoas que são importantes para mim". Os valores obtidos com o alfa de Cronbach $(0,89)$ e os coeficientes de Spearman-Brown $(0,85)$ e de Guttman $(0,84$, sendo a correlação entre metades igual a 0,73 ), são, na generalidade, elevados, indicando a existência de uma boa consistência interna. Por outro lado, também foi encontrada uma boa estabilidade temporal (Teste-Reteste) para a versão portuguesa da escala, uma vez que os coeficientes de correlação de Spearman, entre cada item, foram todos altamente significativos (Variação $=0,354-0,676$ ) e o coeficiente de correlação de Pearson, entre a população total teste-reteste, foi igual a 0,854 ( $[p=0,000] ;$ M. J. Soares et al., 2003).

Vinculação. Para avaliar a vinculação, foi usado o Inventory of Parent and Peer Attachment ([IPPA], Armsden \& Greenberg, 1987; adaptação de Neves et al., 1999). Este instrumento é constituído por três sub-escalas de 25 itens, que são cotadas de forma independente, formando um total de 75 itens, distribuídos equitativamente pela figura materna, paterna e pelos amigos. $\mathrm{O}$ indivíduo responde aos itens numa escala tipo Likert de 5 pontos, sendo que 1 corresponde a "nunca ou quase nunca" e 5 corresponde a "sempre ou quase sempre". O IPPA permite a obtenção de dois tipos de vinculação para cada subescala: (a) Vinculação Segura e (b) Vinculação Insegura (Faria, 2008), sendo que os seus itens enfocam 3 dimensões: Confiança, Comunicação e Alienação. Segundo o processo de adaptação do IPPA para a população portuguesa (Neves et al., 1999), os indivíduos podem ser classificados como tendo um estilo de vinculação seguro ou inseguro, consoante se encontrarem acima ou abaixo do score da mediana. Cunha et al. (2008) refere que ao seguir este procedimento e atendendo ao score da mediana do total da amostra, é possível classificar os participantes deste estudo, como tendo um padrão de vinculação seguro ou inseguro, relativamente à mãe e ao pai.

Os coeficientes de alfa de Cronbach relativos às suas escalas foram elevados, $0,92,0,95$ e 0,93 , respectivamente para as escalas Mãe, Pai e Amigos. Estes valores são indicativos de uma boa consistência interna nas três escalas (Neves et al., 1999). No presente estudo, serão utilizadas as subescalas Pai e Mãe (excluindo-se a escala referente aos amigos), compostas por 50 itens distribuídos equitativamente pela figura materna e paterna, uma vez que pretendemos conhecer a percepção dos participantes sobre as relações de vinculação com os pais.

\section{Procedimento}

Previamente à recolha de dados, procedeu-se ao envio de um pedido de autorização aos presidentes do
Conselho Directivo das escolas e faculdades, para realizar o estudo nos respectivos estabelecimentos de ensino. Procedeu-se, igualmente, ao envio de um pedido de autorização aos pais dos alunos com 17 anos (menores de idade).

Durante a recolha de dados, todos os participantes foram informados acerca dos objectivos do estudo e dos direitos que lhes assistiam, enquanto membros participantes da investigação, através do Consentimento Informado. Os instrumentos foram aplicados durante o ano lectivo de 2008/09, em contexto de sala de aula. A ordem dos questionários foi invertida aleatoriamente com o intuito de evitar o enviesamento dos resultados, causado pelo cansaço e sequência dos instrumentos. Após a recolha de dados, e, dada a natureza correlacional do estudo, estes foram submetidos a um tratamento estatístico, com recurso ao software SPSS, versão 17.

\section{Resultados}

Os resultados estão descritos em duas fases distintas, com base nos dois objectivos principais do presente estudo.

Diferenças entre as Representações de Vinculação e as Dimensões Adaptativas e Não-Adaptativas, Formuladas por Frost et al. (1990) e Hewitt e Flett (1991)

Verificamos que $50,9 \%$ dos participantes apresenta um estilo de Vinculação Seguro para com a Mãe e 47,8\% um estilo de Vinculação Seguro ao Pai. Seguidamente, procedemos à realização do Teste $t$-student, de forma a verificar se existem diferenças estatisticamente significativas entre os padrões de vinculação e as dimensões adaptativas e não-adaptativas de perfeccionismo, formuladas por Frost et al. (1990) e Hewitt e Flett (1991; cfr. Tabela 1). O cálculo do Teste $t$-student entre as dimensões da MPS-H, MPS-F e os padrões de vinculação (pai e mãe) do IPPA revelou que os participantes que possuem uma representação de Vinculação Insegura (pai) apresentam valores médios mais elevados nas dimensões de perfeccionismo: DSPA $(M=11,70 ; D P=2,97 ; p=0,000)$, $\mathrm{EP}(M=11,24 ; D P=3,73 ; p=0,016), \mathrm{e}, \mathrm{CP}(M=11,95$; $D P=3,66 ; p=0,000)$. Os participantes que possuem uma representação de Vinculação Segura (pai) revelam valores médios mais elevados na dimensão $\mathrm{O}(M=23,81$; $D P=4,75 ; p=0,001$ ).

Os participantes com uma representação de Vinculação Insegura (mãe) apresentam valores médios mais elevados nas dimensões de perfeccionismo: DSPA $(M=11,65$; $D P=2,91 ; p=0,000)$, PCE $(M=29,36 ; D P=7,09 ; p=$ $0,017), \mathrm{EP}(M=11,63 ; D P=3,69 ; p=0,000), \mathrm{e}, \mathrm{CP}(M=$ $12,12 ; D P=3,61 ; p=0,000)$. Por sua vez, os participantes que apresentam uma representação de Vinculação Segura (mãe), revelam valores médios mais elevados nas dimensões $\mathrm{O}(M=23,93 ; D P=4,62 ; p=0,000)$ e PAD $(M=79,16 ; D P=12,89 ; p=0,028)$. 
Tabela 1

Valores Médios das Dimensões de Perfeccionismo de Frost et al. (1990) e Hewitt e Flett (1991), em Função do Tipo de Vinculação dos Participantes, Relativamente à Figura Materna e Paterna

\begin{tabular}{|c|c|c|c|c|c|c|}
\hline MPS-F/ MPS-H & Vinculação - Pai & $N$ & $M$ & $D P$ & $t$ & $p$ (2-tailed $)$ \\
\hline \multirow[t]{2}{*}{ DSPA } & Segura & 327 & 10,69 & 3,14 & \multirow[t]{2}{*}{$-4,139$} & \multirow[t]{2}{*}{$0,000 * *$} \\
\hline & Insegura & 305 & 11,70 & 2,97 & & \\
\hline \multirow[t]{2}{*}{ EP } & Segura & 328 & 10,54 & 3,56 & \multirow[t]{2}{*}{$-2,415$} & \multirow[t]{2}{*}{$0,016^{*}$} \\
\hline & Insegura & 304 & 11,24 & 3,73 & & \\
\hline \multirow[t]{2}{*}{$\mathrm{CP}$} & Segura & 324 & 9,67 & 3,08 & \multirow[t]{2}{*}{$-8,406$} & \multirow[t]{2}{*}{$0,000^{* *}$} \\
\hline & Insegura & 302 & 11,95 & 3,66 & & \\
\hline \multirow[t]{2}{*}{$\mathrm{O}$} & Segura & 327 & 23,81 & 4,75 & \multirow[t]{2}{*}{3,228} & \multirow[t]{2}{*}{$0,001 * *$} \\
\hline & Insegura & 307 & 22,52 & 5,27 & & \\
\hline \multirow{3}{*}{ DSPA } & Vinculação - Mãe & & & & \multirow{3}{*}{$-3,804$} & \multirow{3}{*}{$0,000 * *$} \\
\hline & Segura & 347 & 10,75 & 3,13 & & \\
\hline & Insegura & 308 & 11,65 & 2,92 & & \\
\hline \multirow[t]{2}{*}{ PCE } & Segura & 344 & 28,09 & 6,47 & \multirow{2}{*}{$-2,394$} & \multirow[t]{2}{*}{$0,017^{*}$} \\
\hline & Insegura & 303 & 29,36 & 7,09 & & \\
\hline \multirow[t]{2}{*}{ EP } & Segura & 348 & 10,18 & 3,52 & \multirow{2}{*}{$-5,151$} & \multirow{2}{*}{$0,000^{* *}$} \\
\hline & Insegura & 307 & 11,63 & 3,69 & & \\
\hline \multirow[t]{2}{*}{$\mathrm{CP}$} & Segura & 343 & 9,58 & 3,09 & \multirow[t]{2}{*}{$-9,578$} & \multirow[t]{2}{*}{$0,000^{* *}$} \\
\hline & Insegura & 306 & 12,12 & 3,61 & & \\
\hline
\end{tabular}

Relação entre os Factores Confiança, Comunicação e Alienação, com as Dimensões de Perfeccionismo, de Frost et al. (1990) e Hewitt e Flett (1991)

Com o intuito de percebermos qual o tipo de relação existente entre os três factores das sub-escalas Pai e Mãe do IPPA (Confiança, Comunicação e Alienação) e as dimensões de perfeccionismo do MPS-H e do MPS-F, procedemos à realização do Coeficiente de Correlação de Pearson. O cálculo do coeficiente de correlação de Pearson entre as dimensões do MPS-H e os factores do IPPA revela a existência de correlações significativas para um nível de significância de 0,05 e 0,01 $(p<0,05$ e $p<0,01)$. Relativamente ao factor Confiança, verificamos que, enquanto que na sub-escala Pai existe uma correlação negativa $(r=-0,12 ; p=0,003)$, estatisticamente significativa para um nível de significância de $0,01(p<0,01)$, entre as variáveis Confiança ao Pai e Perfeccionismo Dirigido aos Outros (PDO); na sub-escala Mãe, existe uma correlação negativa, estatisticamente significativa $(r$ $=-0,12 ; p=0,002)$, entre as variáveis Confiança à Mãe $\mathrm{e}$ o Perfeccionismo Socialmente Prescrito (PSP). No que concerne ao factor Comunicação, verificamos que, na sub-escala Pai, existe uma correlação positiva, estatisticamente significativa $(r=0,12 ; p=0,002)$, entre as variá- veis Comunicação ao Pai e o Perfeccionismo Auto-Dirigido (PAD), ainda na sub-escala Pai, concluímos a existência de uma correlação negativa, estatisticamente significativa $(r=-0,12 ; p=0,003)$, entre o factor Comunicação e a dimensão Perfeccionismo Dirigido aos Outros. Relativamente à sub-escala Mãe, podemos igualmente verificar a existência de uma correlação positiva, estatisticamente significativa $(r=0,14 ; p=0,000)$, entre o factor Comunicação à Mãe e a dimensão Perfeccionismo AutoDirigido, e, uma correlação negativa, estatisticamente significativa $(r=-0,08 ; p=0,047)$, entre o factor Comunicação e o Perfeccionismo Dirigido aos Outros. Por último, relativamente ao factor Alienação, podemos inferir que, na sub-escala Mãe, existe uma correlação positiva, estatisticamente significativa $(r=0,09 ; p=0,027)$, entre o factor Alienação à mãe e a dimensão PAD, e, uma correlação negativa, estatisticamente significativa $(r=-0,08$; $p=0,041$ ), entre o mesmo factor (Alienação à Mãe) e a dimensão PDO.

O cálculo do coeficiente de correlação de Pearson entre as dimensões do MPS-F e os factores do IPPA evidencia correlações estatisticamente significativas, para um nível de significância de $0,05(p<0,05)$ e $0,01(p<0,01)$. No que respeita ao factor Confiança (Pai e Mãe), verificamos a existência de correlações negativas, estatistica- 
mente significativas, de magnitude moderada, entre as variáveis Confiança ao Pai e à Mãe e a dimensão Críticas Parentais ([CP]; CF Pai: $r=-0,43 ; p<0,000$; CF Mãe: $r$ $=-0,52 ; p<0,000)$. Verificamos também a existência de uma correlação negativa, estatisticamente significativa, de magnitude fraca, entre as variáveis Confiança à Mãe e ao Pai e a dimensão Dúvidas Sobre as Próprias Acções ([DSPA]; CF Pai: $r=-0,21 ; p=0,000$; CF Mãe: $r=$ $0,22 ; p=0,000)$.

Observamos a existência de correlações negativas, estatisticamente significativas, de magnitude fraca, entre as variáveis Comunicação ao Pai e à Mãe e a dimensão CP (CM Pai: $r=-0,32 ; p<0,000$; CM Mãe: $r=-0,38$; $p<0,000$ ). Por último, existe uma correlação negativa, estatisticamente significativa, de magnitude fraca, entre as variáveis Confiança à Mãe e Expectativas Parentais ([EP]; $r=-0,28 ; p<0,000$ ) e uma correlação positiva, estatisticamente significativa, de magnitude igualmente fraca, entre o factor Comunicação com a Mãe e a dimensão Organização ([O]; $r=0,22 ; p<0,000)$.

Uma vez concretizados os objectivos propostos, procurámos perceber se existem diferenças entre géneros, quanto às representações de vinculação (Segura ou Insegura) e às dimensões de perfeccionismo. Inicialmente, procedemos à realização do Teste do Qui-quadrado, para averiguar se existiam diferenças estatisticamente significativas entre géneros, relativamente ao tipo de representação de vinculação à mãe e ao pai. No que concerne a vinculação ao pai, verificámos que $53,3 \%$ dos participantes do género masculino (filhos) apresentavam uma Vinculação Segura ao pai e que $46,7 \%$ apresentava uma vinculação insegura. Quanto aos participantes do género feminino (filhas), 50,9\% revelaram ter uma Vinculação Segura ao pai e 49,1\% uma Vinculação Insegura. No que respeita à vinculação à mãe, $50,4 \%$ dos participantes do género masculino apresentavam uma Vinculação Segura à mãe e 49,6\% uma Vinculação Insegura. Por seu turno, $54,5 \%$ dos participantes do género feminino demonstraram uma Vinculação Segura à mãe e 45,5\% uma Vinculação Insegura à mesma. Contudo estes resultados não são estatisticamente significativos, não podendo comprovar a existência de diferenças entre géneros, no que concerne ao tipo de representação de vinculação, em relação à figura materna e paterna.

Por último, de forma a verificar se existiam diferenças significativas nas dimensões de perfeccionismo, em função do género dos sujeitos, efectuámos o teste $t$-student para comparação de duas médias (design entre-sujeitos). Foi possível observar que as dimensões Expectativas Parentais (EP) e Organização (O) evidenciam diferenças estatisticamente significativas entre géneros. No que concerne a dimensão EP, constatamos que os participantes do género masculino apresentam valores médios significativamente mais elevados $(M=11,24 ; D P=3,497 ; p=$ 0,036 ), comparativamente com os participantes do género feminino. Inversamente, na dimensão Organização, os participantes do género feminino revelam valores médios significativamente mais elevados $(M=23,92 ; D P=4,747$; $p=0,000$ ), para um nível de significância de 0,05 e 0,01 $(p<0,05$ e $p<0,01)$, comparativamente com os sujeitos do género masculino.

\section{Discussão}

A principal finalidade da presente investigação foi estudar as relações entre os níveis de perfeccionismo e as representações de vinculação parental em jovens adultos e averiguar a existência de uma relação entre as representações de vinculação e as diferentes dimensões de perfeccionismo. Os resultados do presente estudo apontam para a existência de uma relação entre o tipo de representações de vinculação (Seguro ou Inseguro) e a manifestação de alguns traços de perfeccionismo maladaptativos ou adaptativos.

Este estudo sugere que os sujeitos com uma representação de Vinculação Insegura à mãe e ao pai tendem a apresentar valores médios mais elevados em dimensões maladaptativas de perfeccionismo (Dúvidas Sobre as Próprias Acções, Preocupação em Cometer Erros, Expectativas Parentais e Críticas Parentais). Relativamente às representações de uma Vinculação Segura, os resultados parecem também indicar que se relacionam com dimensões adaptativas de perfeccionismo, o Perfeccionismo Auto-Dirigido e a Organização, dimensão esta última que, apesar de possuir uma correlação mais fraca relativamente ao valor total do perfeccionismo, é considerada como sendo um factor de protecção, em relação ao desenvolvimento de perfeccionismo patológico ou maladaptativo (Cruz, 2009).

Os resultados indicam que a combinação das dimensões Expectativas Parentais elevadas e Críticas Parentais elevadas podem ter efeitos maladaptativos no traço de perfeccionismo, condicionando a sua representação de vinculação. No mesmo sentido, Rice et al. (2005), concluem que a combinação de Expectativas Parentais Elevadas e Críticas Parentais elevadas predizem o perfeccionismo maladaptativo. Rice et al. (1996) fornecem ainda evidências empíricas que suportam a hipótese de que pais de perfeccionistas maladaptativos são caracterizados como sendo severos, críticos, controladores e exigentes, estabelecendo padrões exorbitantemente elevados para os seus filhos.

Relativamente às diferenças entre géneros nas dimensões de perfeccionismo, foi possível verificar que os filhos apresentam valores mais elevados na dimensão Expectativas Parentais, enquanto as filhas apresentam valores mais elevados na dimensão Organização. Podemos concluir que os resultados relativos à dimensão Expectativas Parentais corroboram as conclusões apresentadas por Gamble e Roberts (2005). Estes autores salientam que os pais revelam possuir maiores expectativas e maiores aspirações para os seus filhos, incentivando-os a atingir maiores objectivos, do que para com as suas filhas, o que poderá explicar a obtenção de valores médios mais ele- 
vados na dimensão Expectativas Parentais, por parte dos filhos homens, tal como observado no nosso estudo. Relativamente aos resultados obtidos na dimensão Organização, não encontrámos estudos que tenham observado resultados semelhantes nesta dimensão de perfeccionismo, em amostras com as mesmas características da nossa amostra.

No que concerne à relação entre os três factores do IPPA (Confiança, Comunicação e Alienação) e as dimensões de perfeccionismo de Frost et al. (1990) e Hewitt e Flett (1991), podemos concluir que os resultados obtidos, apesar apresentarem associações fracas e moderadas, sugerem a existência de uma relação positiva entre os factores Confiança e Comunicação (característicos de uma Vinculação Segura) e as dimensões adaptativas de perfeccionismo, PP e O (Frost et al, 1990); e o PAD (Hewitt \& Flett, 1991). Concomitantemente, os mesmos factores possuem uma relação negativa com as dimensões maladaptativas de perfeccionismo DSPA, PCE, EP e CP (Frost et al., 1990) e PSP (Hewitt \& Flett, 1991). Estes resultados parecem reforçar os pressupostos teóricos e empíricos, descritos na literatura, de que o desenvolvimento do perfeccionismo poderá ser um produto das interacções das crianças com os seus pais (Barrow \& Moore, 1983; Burns, 1980; Kawamura et al., 2002; Rice et al., 2005) e que as representações de uma vinculação segura estão relacionadas com a manifestação de perfeccionismo adaptativo, assim como as representações de uma vinculação insegura estão relacionadas com a manifestação de perfeccionismo maladaptativo (Craddock et al., 2008; Perris \& Anderson, 2000; Rice \& Mirzadeh, 2000).

O presente estudo também revelou a existência de correlações negativas entre o factor Alienação (factor associado a uma representação de vinculação insegura) e algumas dimensões maladaptativas de perfeccionismo. No mesmo sentido, Rice e Mirzadeh (2000) concluem que a qualidade de vinculação é um preditor imperfeito de perfeccionismo maladaptativo, tendo estes autores verificado que alguns estudantes, classificados com uma Vinculação Segura, possuíam perfeccionismo maladaptativo, advertindo, também, para o facto de que as associações entre a vinculação e perfeccionismo devem ser cautelosamente interpretadas, dada a natureza correlacional e de auto-relato dos dados utilizados.

De futuro, seria importante continuar a investir nesta área de investigação, com o intuito de aprofundar o conhecimento sobre os factores que contribuem para a origem do perfeccionismo. O presente estudo pode promover a intervenção precoce junto das famílias que, através das suas práticas parentais e experiências relacionais disfuncionais, constituem um factor de vulnerabilidade para o desenvolvimento do perfeccionismo maladaptativo. Assim como, numa perspectiva psico-educacional, promover uma intervenção psicoterapêutica, direccionada para indivíduos com elevados níveis de perfeccionismo.

Rice e Mirzadeh (2000) referem que, sob um ponto de vista educacional, seria importante ajudar o indivíduo a aprender sobre o perfeccionismo e a fortalecer as dimensões adaptativas deste traço de personalidade (e.g., Padrões Pessoais e Organização), enquanto se questiona a utilidade do perfeccionismo maladaptativo. Concomitantemente, os autores revelam que a teoria da vinculação poderá ser uma componente importante da intervenção, por parte do terapeuta, junto de indivíduos perfeccionistas, uma vez que uma das possíveis razões para que o perfeccionismo maladaptativo seja tão resistente à mudança, poderá ser o facto de que "mudar o perfeccionismo requer mudanças profundas nas percepções sobre o self $\mathrm{e}$ os outros, o que, por sua vez, requer mudanças fundamentais nas experiências relacionais" (Rice \& Mirzadeh, 2000 , p. 249). Em suma, os terapeutas poderão auxiliar os seus pacientes a obter um maior insight sobre as influências desenvolvimentais do seu perfeccionismo, através da exploração das suas representações vinculativas e do papel da sua família na modelagem e manutenção das suas tendências perfeccionistas.

No que concerne as limitações deste estudo, podemos referir que estão relacionadas com os instrumentos de avaliação seleccionados. Uma vez que os instrumentos seleccionados são questionários de auto-relato, existe a possibilidade que factores como a desejabilidade social, o estilo de resposta por extremos e a reconstrução variável sobre o que aconteceu no passado, baseada em circunstâncias de vida actuais (Laghi et al., 2009), possam ter contribuído para uma distorção dos resultados. Halverson (1988) afirma que, sem outro tipo de fontes de dados, não possuímos qualquer tipo de informação sobre a acuidade do relato do sujeito e, assim sendo, devemos interpretar os dados recolhidos de uma forma contemporânea e não longitudinal.

O IPPA é um instrumento que apresenta algumas limitações, uma vez que, por não possuir normas para a interpretação de resultados, não permite determinar com precisão o tipo de vinculação insegura dos sujeitos. Segundo Rice e Mirzadeh (2000), alguns tipos de vinculação insegura podem ser mais relevantes para o desenvolvimento de perfeccionismo, do que outros (Rice et al., 2005; Wei et al., 2004). Neste sentido, sugerimos a utilização de instrumentos como a AAI (Adult Attachment Interview), que parece colmatar estas limitações.

Por último, consideramos importante a realização de um estudo longitudinal, de forma a avaliar a vinculação e a manifestação de traços de perfeccionismo, ao longo da vida.

Rice e Mirzadeh (2000) reforçam a fraca capacidade que existe para prever o desenvolvimento de perfeccionismo maladaptativo, através da vinculação. Como foi referido na revisão da literatura, parcas são as investigações empíricas sobre o perfeccionismo e a vinculação (Wei et al., 2004). Esta é uma temática recente, que requer um maior investimento e aprofundamento sobre a existência de outras variáveis (e.g., estilos parentais, acontecimentos de vida negativos, satisfação parental) que, em conjunto com uma representação de vinculação insegura, contribuam para o desenvolvimento de perfeccio- 
Oliveira, D. F., Carmo, C., José Cruz, P. \& Brás, M. (2012). Perfeccionismo e Representação Vinculativa em Jovens Adultos.

nismo maladaptativo.

\section{Referências}

Adkins, K. K., \& Parker, W. (1996). Perfectionism and suicidal preoccupation. Journal of Personality, 64, 529-543.

Armsden, G., \& Greenberg, M. (1987). The inventory of parent and peer attachment: Mother, father, and peer attachment (Rev. Ed.). Washington, DC: Department of Psychology, University of Washington.

Azevedo, M., Bos, S., Soares, M., Pereira, A., Marques, M., Maia, B., et al. (2008). Perfectionism and sleep disturbance: A longitudinal study. Journal of Sleep Research, 17, 258-258.

Bardone-Cone, A. M., Sturm, K., Lawson, M. A., Robinson, D. P., \& Smith, R. (2010). Perfectionism across stages of recovery from eating disorders. International Journal of Eating Disorders, 43(2), 139-148.

Barrow, J., \& Moore, C. (1983). Group interventions with perfectionistic thinking. Personnel and Guidance Journal, 61, 612-615.

Blatt, S. J. (1995). The destructiveness of perfectionism: Implications for the treatment of depression. American Psychologist, 50(12), 1003-1020.

Bowlby, J. (1977). The making and breaking of affectional bonds: 1. Aetiology and Psychopathology in the light of attachment theory. British Journal of Psychiatry, 130, 201210.

Burns, D. (1980, November). The perfectionist's script for selfdefeat. Psychology Today, 14(11), 34-51.

Camadan, F. (2010). Predicting the student's perfectionism from their parents' perfectionism. Procedia Social and Behavioral Sciences, 2, 4260-4265.

Chang, E. C. (1998). Cultural differences, perfectionism, and suicidal risk in a college population: Does social problem solving still matter? Cognitive Therapy and Research, 22, 237-254.

Chang, E. C. (2002). Examining the link between perfectionism and psychological maladjustment: Social problem solving as a buffer. Cognitive Therapy and Research, 26, 581-595.

Chang, E. C., \& Sanna, L. J. (2001). Negative attributional style as a moderator of the link between perfectionism and depressive symptoms: Preliminary evidence for an integrative model. Journal of Counseling and Psychology, 48, 490-495.

Craddock, A. E., Church, W., \& Sands, A. (2008). Family of origin characteristics as predictors of perfectionism. Australian Journal of Psychology, 1, 1-9.

Cruz, J. P. (2009). Perfeccionismo, saúde e doenças. In J. P. Cruz, S. N. Jesus, \& C. Nunes (Eds.), Bem-estar e qualidade de vida: Contributos da Psicologia da Saúde (pp. 127-160). Alcochete, Portugal: Textiverso.

Cunha, M., Soares, I., \& Pinto-Gouveia, J. (2008). The role of individual temperament, family and peers in social anxiety disorder: A controlled study. International Journal of Clinical and Health Psychology, 8(3), 631-655.

Enns, M. W., Cox, B. F., Sareen, F., \& Freeman, P. (2001). Adaptive and maladaptive perfectionism in medical students: A longitudinal investigation. Medical Education, 35, 10341042 .

Faria, C. M. G. M. (2008). Vinculação e desenvolvimento epistemológico em jovens adultos. (Tese de Doutorado nãopublicada). Universidade do Minho, Braga, Portugal.

Frost, R. O., Lahart, C. M., \& Rosenblate, R. (1991). The development of perfectionism: A Study of Daughters and
Their Parents. Cognitive Therapy and Research, 15(6), 469489.

Frost, R. O., Marten, P., Lahart, C. M., \& Rosenblate, R. (1990). The dimensions of perfectionism. Cognitive Therapy and Research, 14, 449-468.

Gamble, S. A., \& Roberts, J. E. (2005). Adolescents' perceptions of primary caregivers and cognitive style: The roles of attachment security and gender. Cognitive Therapy and Research, 29(2), 123-141.

Geada, M. (1990). Padrões de vinculação afectiva e níveis de desenvolvimento do auto-conhecimento em toxicodependentes e não-toxicodependentes. Jornal de Psicologia, 9(4/5), 14-18.

Gomes, V. F., \& Bosa, C. A. (2010). Representações mentais de apego e percepção de práticas parentais por jovens adultas. Psicologia: Reflexão e Crítica, 23(1), 11-18.

Halverson, C. F. (1988). Remembering your parents: Reflections on the retrospective method. Journal of Personality, 56(2), 435-442.

Hamachek, D. E. (1978). Psychodynamics of normal and neurotic perfectionism. Psychology: A Journal of Human Behavior, 15(1), 27-33.

Hewitt, P. L., \& Flett, G. L. (1990). Perfectionism and depression: A multidimensional analysis. Journal of Social Behavior and Personality, 5, 423-438.

Hewitt, P. L., \& Flett, G. L. (1991). Perfectionism in the self and social contexts: Conceptualization, assessment and association with psychopathology. Journal of Personality and Social Psychology, 60, 456-470.

Kawamura, K. Y., Frost, R. O., \& Harmatz, M. G. (2002). The relationship of perceived parenting styles to perfectionism. Personality and Individual Differences, 32, 317-327.

Laghi, F., D'Alessio, M., Pallini, S., \& Baiocco, R. (2009). Attachment representations and time perspective in adolescence. Social Indicators Research, 90, 181-194.

Mota, C. P., \& Matos, P. M. (2009). Apego, conflito e autoestima em adolescentes de famílias intactas e divorciadas. Psicologia: Reflexão e Crítica, 22(3), 344-352.

Neves, L., Soares, I., \& Silva, C. (1999). Inventário da Vinculação na Adolescência (IPPA). In L. Almeida, M. Gonçalves, \& M. Simões (Eds.), Testes e Provas Psicológicas em Portugal (pp. 37-48). Braga, Portugal: Associação dos Psicólogos Portugueses.

Pais-Ribeiro, J. L. (2008). Metodologia de investigação em Psicologia e Saúde. Porto, Portugal: Legis.

Perris, C., \& Anderson, P. (2000). Experiences of parental rearing and patterns of attachment in adulthood. Clinical Psychology and Psychotherapy, 7, 279-288.

Platts, H., Tyson, M., \& Mason, O. (2002). Adult attachment style and core beliefs: Are they linked?. Clinical Psychology and Psychotherapy, 9, 332-348.

Rice, K. G., Ashby, J. S., \& Preusser, K. J. (1996). Perfectionism, relationships with parents, and self-esteem. Individual Psychology, 52(3), 246-260.

Rice, K. G., Lopez, F. G., \& Vergara, D. (2005). Parental/Social influences on perfectionism and adult attachment orientations. Journal of Social and Clinical Psychology, 24(4), 580-605.

Rice, K. G., \& Mirzadeh, S. A. (2000). Perfectionism, attachment, and adjustment. Journal of Counseling Psychology, 47, 238-250.

Shafran, R., \& Mansell, W. (2001). Perfectionism and Psychopathology: A review of research and treatment. Clinical Psychology Review, 21, 879-906.

Sherry, S. B., Hewitt, P. L., Flett, G. L., \& Harvey, M. (2003). Perfectionism dimensions, perfectionistic attitudes, dependent 
attitudes, and depression in psychiatric patients and university students. Journal of Counseling Psychology, 50(3), 373-386.

Soares, M. J., Gomes, A. A., Macedo, A. F., \& Azevedo, M. H. P. (2003). Escala Multidimensional de Perfeccionismo: Adaptação à população portuguesa. Revista Portuguesa de Psicossomática, 5(1), 46-55.

Sochos, A., \& Tsalta, A. (2008). Depressiogenic cognition and insecure attachment: A motivational hypothesis. International Journal of Psychology and Psychological Therapy, 8(2), 157-170.

Soenens, B., Vansteenkiste, M., Luyten, P., Duriez, B., \& Goossens, L. (2005). Maladaptive perfectionistic self-representations: The meditational link between psychological control and adjustment. Personality and Individual Differences, 38, 487-498.

Stoeber, J., Feast, A. R., \& Hayward, J. A. (2009). Self-oriented and socially prescribed perfectionism: Differential relationships with intrinsic and extrinsic motivation and test anxiety. Personality and Individual Differences, 47(5), 423-428.

Wei, M., Mallinckrodt, B., Russel, D. W., \& Abraham, W. T. (2004). Maladaptive perfectionism as a mediator and moderator between Adult attachment and depressive mood. Journal of Counseling Psychology, 51(2), 201-212.

Wu, K. D., \& Cortesi, G. T. (2009). Relations between perfectionism and obsessive-compulsive symptoms: Examination of specificity among the dimensions. Journal of Anxiety Disorders, 23(3), 393-400. 